\title{
Development, integration and operation of mobile, Android-based medical devices in hospitals: Experiences from the GlucoTab® system
}

\author{
Stephan Spat", Kevin Theuermann" ${ }^{\#}$, Bernhard Höll ${ }^{\#}$, Peter Beck ${ }^{\#}$, Thomas R. Pieber, ${ }^{\#, *}$ \\ ${ }^{\#}$ HEALTH - Institute for Biomedicine and Health Sciences \\ JOANNEUM RESEARCH Forschungsgesellschaft mbH, Graz, Austria \\ ${ }^{*}$ Division of Endocrinology and Metabolism, Department of Internal Medicine, \\ Medical University of Graz, Graz, Austria
}

\begin{abstract}
- the use of mobile applications is increasing in the hospitals. Besides numerous advantages such as time- and location independent view of examination results, documentation of therapy at the patient site, point of care workflow and decision support, smartphone- or tablet-based mobile systems pose challenges to system manufacturers and hospital-IT operators. In this paper we present our experiences on managing mobile, Android-based medical devices in hospitals based on the GlucoTab ${ }^{\circledR}$. GlucoTab ${ }^{\circledR}$ is a client-server system using a Google Android-based tablet as a frontend device for diabetes workflow and insulin decision support in hospitals. We analysed the hospital IT-infrastructure for mobile devices in eight different hospitals and based on recent published reports. Based on this analysis we developed suggestions on how to handle typical administrative issues like domain integration, software inventory and distribution or user management. Depending on the integration prerequisites of hospitals we finally provided five different integration scenarios for the GlucoTab ${ }^{\circledR}$ system.
\end{abstract}

Keywords - mobile, clinical decision support, diabetes mellitus type 2, hospital ward, medical device

\section{INTRODUCTION}

The use of mobile applications in the private and public sector increases constantly [1]. In the hospital environment, mobile devices provide numerous advantages compared to stationary computers or mobile ward trolleys. For example, examination results can be viewed by healthcare professionals (HP) independent of time- and location by using smartphones or tablets which are easy to carry. Documentation of medical therapy can be performed directly at the patient site. Mobile decision support systems provide additional value to HP by structuring the treatment workflow and automatically suggesting the optimal medication at the point of care [2]-[4]. Beside all these advantages, smartphone- or tablet-based mobile systems pose challenges to system manufacturers and hospital-IT operators in terms of development, integration, operation and maintenance in the hospital environment, especially for systems not based on standard Windows operating systems. If the mobile application is used as a medical device other challenges like improved requirements on software quality and risk assessment have to be addressed. The present work focuses on suggestions how to manage mobile, Android-based medical devices in hospitals based on the experiences of the GlucoTab® system.

\section{METHODS}

\section{A. The GlucoTab ${ }^{\circledR}$ System}

The GlucoTab ${ }^{\circledR}$ system is a mobile, client-server system with a Google Android-based tablet acting as a frontend device for clinical decision support (Fig. 1). GlucoTab ${ }^{\circledR}$ supports glycemic management for patients with type 2 diabetes mellitus in the hospital. Automatic insulin dosing is provided based on blood glucose spot measurements in the morning, at midday, in the evening and at night. Main users are nurses and doctors who are responsible for diabetes therapy at the ward. GlucoTab ${ }^{\circledR}$ is a class I medical device and has been developed according to medical software standards. Each user has a personal account with username and password to login to the system. Details about the development and the functions of the GlucoTab system can be found in [4]-[6].

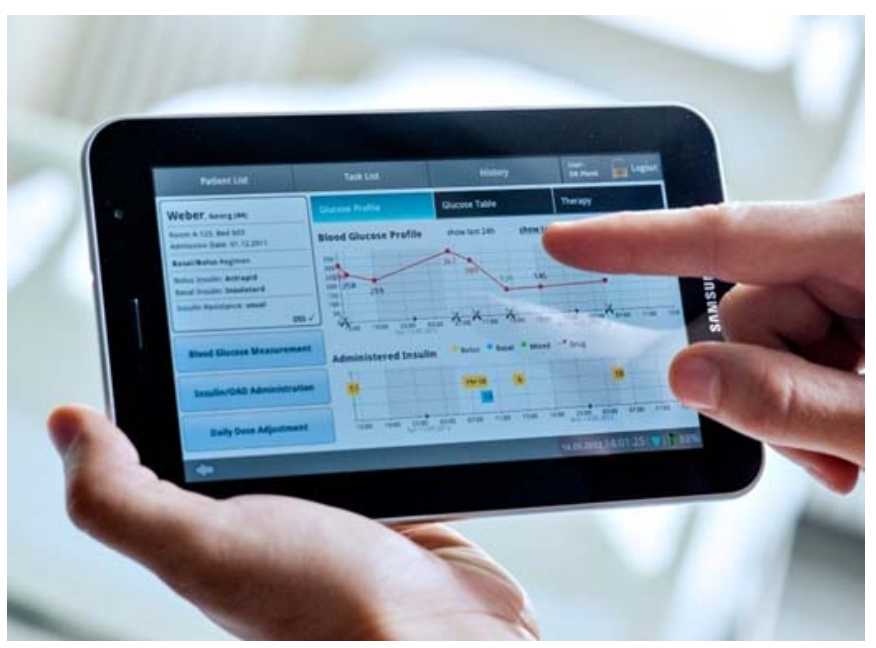

Fig 1 The main screen of a Samsung Galaxy Tab 7” running the GlucoTab ${ }^{\circledR}$ system which provides information about blood glucose control and insulin injections 


\section{B. Clinical Trials and Hospital IT Infrastructure}

Two clinical trials were performed at the Medical University of Graz, involving four different wards (endocrinology, cardiology, plastic surgery and nephrology). We installed the GlucoTab® backend on a virtualized server environment, operated by the hospital IT-department. The server was built using a Java Enterprise Open Source stack running on Apache Tomcat. We used two to three Samsung Galaxy Tab 7” per ward as frontend devices. The mobile client devices running the GlucoTab® frontend App communicated via Wi-Fi with the backend server. Approximately $80 \mathrm{HP}$ used the GlucoTab® system during two clinical trials, and in total 129 patients were treated with the GlucoTab ${ }^{\circledR}$.

Preliminary results of the clinical trials are promising. Blood glucose could be titrated in the desired target range of 140 to $180 \mathrm{mg} / \mathrm{dl}$. Users felt confident using the system and no GlucoTab®-related adverse event could be detected. Usability testing indicates that the system is well accepted by HP.

In this work we focused on the evaluated the existing IT infrastructure of five Austrian, one Danish and one German hospital using an online questionnaire and personal interviews. The German hospital already uses an electronic medical record system including a paperless fever chart and a Clinical Provider Order Entry (CPOE). In addition, meetings with the local hospital IT-operator in Graz were organized to discuss the integration of the GlucoTab ${ }^{\circledR}$ system. Based on our experiences from the clinical trials, the collected data as well as the research in relevant literature and the lessons learned from the different phases of the GlucoTab ${ }^{\circledR}$ lifecycle are presented here.

\section{RESULTS}

\section{A. Hospital IT Infrastructure for Mobile Devices}

Wi-Fi access at clinical wards is a fundamental prerequisite for running the GlucoTab® system. Results from the online questionnaire showed that six out of eight (75\%) hospitals provide Wi-Fi in nearly all wards. Two hospitals installed WiFi only at selected wards but none of the hospitals had already integrated a Mobile Device Management System (MDMS).

A recent presentation from the Healthcare Information and Management Systems Society (HIMSS) Europe [7] showed that $91 \%$ of all US hospitals already have installed Wi-Fi. In Spain $85 \%$ and $57 \%$ of German hospitals offer Wi-Fi at their wards. In Italy only $32 \%$ of the hospitals offer Wi-Fi.

HIMSS also reported that $69 \%$ of US hospitals supply clinicians with smart phones and 43\% already implemented tablet computers designed for healthcare use [8]. Questionnaires performed by HIMSS also indicated, that tablet computers are the fastest growing mobile segment in US hospitals and coverage should increase to $63 \%$ in the next years. Please refer to Table 1 for details.

TABLE I

Mobile DeVICES PROVIDED TO CLINICIANS IN US HosPitALS

\begin{tabular}{|l|l|l|}
\hline Mobile Technology & $\begin{array}{l}\text { Current } \\
\text { situation (\%) }\end{array}$ & $\begin{array}{l}\text { Target } \\
\text { situation (\%) }\end{array}$ \\
\hline Laptop computers & 87 & 15 \\
\hline
\end{tabular}

\begin{tabular}{|l|l|l|}
\hline $\begin{array}{l}\text { Computers/ } \\
\text { workstations on wheel }\end{array}$ & 81 & 14 \\
\hline Smart-phones & 69 & 30 \\
\hline Pagers & 67 & 8 \\
\hline Cellular Phones & 56 & 14 \\
\hline $\begin{array}{l}\text { Tablet computers NOT } \\
\text { designed for healthcare }\end{array}$ & 44 & 49 \\
\hline $\begin{array}{l}\text { Tablet computers } \\
\text { designed for healthcare }\end{array}$ & $\mathbf{4 3}$ & $\mathbf{6 3}$ \\
\hline
\end{tabular}

Patient enrolment, transfer and discharge in the GlucoTab® system was performed in synchronization with the hospital patient management system (PMS), which is usually part of the hospital information system (HIS). Integration was achieved using HL7 messages via a HL7 interface engine. Therefore, a HIS providing a HL7 interface engine is an important facilitator for GlucoTab® integration.

All included hospitals had already implemented a HL7 interface engine. Fig 2 shows the diagram of GlucoTab® HL7 communication with the PMS/HIS.

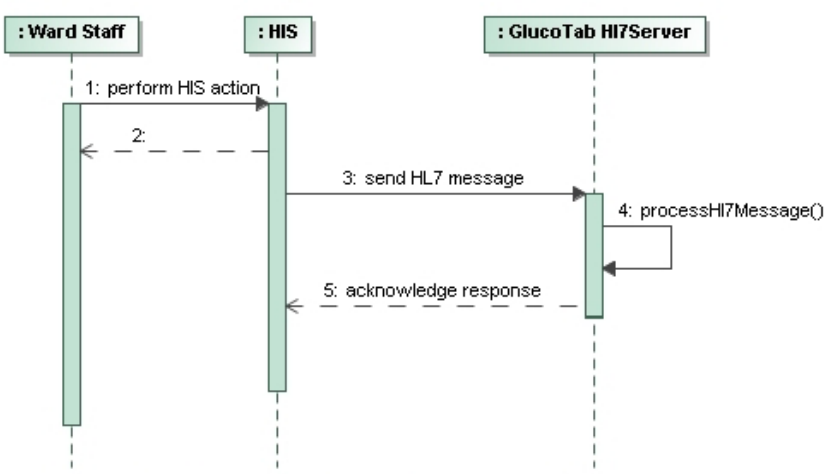

Fig 2 Diagram of the GlucoTab ${ }^{\circledR}$ HL7 communication with the PMS/HIS

\section{B. Software as Medical Devices and Quality Management}

We used an iterative software development process to design and implement the GlucoTab ${ }^{\circledR}$ system. In every iteration, paper or software mock-ups were prepared to gather user feedback in usability tests. Results were discussed in interdisciplinary teams of doctors, nurses and technicians and were fed into the next iteration cycle. We implemented a quality management system (QMS) for medical devices based on relevant standards (IEC 62304, IEC 62366) according to the medical device directive for software and certified the QMS. Consequently, we developed the GlucoTab ${ }^{\circledR}$ software according to these standards within the QMS framework. The introduction of the QMS changed the development process within the team. Risk assessment became a central issue in every step of the development process. For example, the risk of every software change has to be documented and assessed before approval and implementation. Suitable measures (e.g. changes in software design, user training or remarks in user manual) have to be defined to eliminate or at least minimize the potential harm caused by the risk before any line of code can be written. Documentation effort increased tremendously during the development process. We calculated that the introduction of the QMS-extension is equivalent to 
approximately 30 person months. Software development effort doubled due to more extensive testing (unit testing, integration testing, system testing at lab and at ward, usability testing at ward, acceptance testing) and documentation, but first feedback from the clinical trials show that software quality improved due to the more extensive documentation and testing. In addition, clinical validation was performed to prove safety and efficacy of the system.

\section{GlucoTab® Integration, Operation and Maintenance}

In general a hospital which runs the GlucoTab ${ }^{\circledR}$ system provides one backend server and two to three Android-based tablets per ward. A medium sized hospital IT-operator responsible for five technically associated hospitals with five wards each would have to manage at least 50 GlucoTab ${ }^{\circledR}$ client tablets. Thus, the installation and operation of GlucoTab ${ }^{\circledR}$ would challenge the hospital-IT operator in terms of integration, operation and maintenance. The following organisational and technical requirements for a smooth performance of the system within the hospital ITinfrastructure have been identified:

1) Domain Integration: GlucoTab ${ }^{\circledR}$ client tablet devices are Android-based but hospital IT-infrastructure is usually based on Microsoft Windows. It is not possible to adequately integrate Android devices into a Windows Domain.

2) Software Inventory and Distribution: Taking inventory of installed software and automated distribution of new software is a main requirement of a hospital IToperator.

3) User Management and Access Authorization: Shift work and staff turnover result in a high number of HP using the GlucoTab® system. Experiences from the clinical trials show that $80 \mathrm{HP}$ used the GlucoTab ${ }^{\circledR}$ at four different wards during a period of seven months. Nonautomatic user management with software developed inhouse was very time consuming.

4) Remote Maintenance: Remote device access for maintenance was identified as a main requirement for hospital IT-operators.

MDMS like AirWatch ${ }^{1}$ or MobilIron $^{2}$ implement functionality to substitute domain integration, to support automatic software distribution and also to perform remote control. We identified three different mobile device management scenarios for the GlucoTab ${ }^{\circledR}$ in hospitals. In the first scenario (S1) MDMS is directly hosted in the hospital by the hospital IT-operator. Scenario two (S2) assumes that the manufacturer runs the MDMS for the GlucoTab ${ }^{\circledR}$ frontend devices. In scenario three (S3) the GlucoTab ${ }^{\circledR}$ backend provides MDMS functionality directly for features like distribution of GlucoTab ${ }^{\circledR}$ frontend software, distribution of Wi-Fi certificates or lockdown of the Android clients (kiosk mode). S1 offers the advantage that the hospital MDMS also supports other mobile devices in addition to GlucoTab ${ }^{\circledR}$ and no communication interfaces outside the hospital network have to be opened. The whole effort for the GlucoTab ${ }^{\circledR}$ frontend maintenance lies with the hospital IT-operator which may not be desired if the GlucoTab ${ }^{\circledR}$ is the only mobile Android-based device at the hospital. If no MDMS is available in the hospital, the manufacturer of the GlucoTab ${ }^{\circledR}$ system can provide such a system at its premises. In this scenario, the work effort for the hospital IT-operator would be significantly reduced. Digital certificates and possibly other safety critical data will have to be stored in the MDMS hosted by the manufacturer, which requires a certain level of trust from the hospital IT-provider. S3 provides a minimized version of S2. Only absolutely necessary functionality would be provided as part of the GlucoTab ${ }^{\circledR}$ system and no additional system has to be bought, installed and maintained.

For hospital user management and access authorization we suggest interfacing the LDAP or Active Directory system of the hospital in order to avoid manual user management. Please refer to Fig 3.

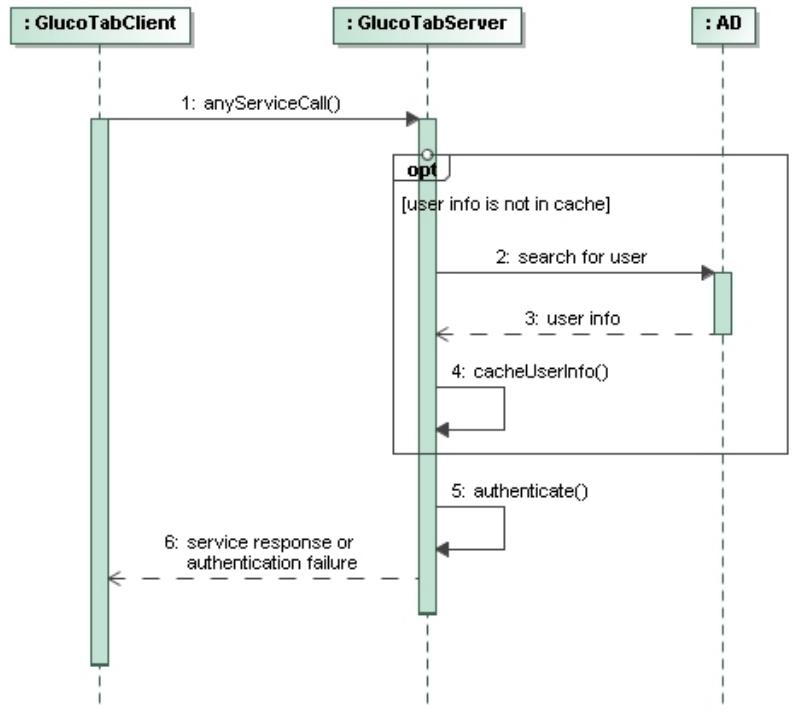

Fig 3 Diagram of the suggested GlucoTab® Active Directory (AD) communication with the patient management service of the hospital

Remote access functionality is standard functionality of some MDMS. Third party tools like $\mathrm{VNC}^{3}$ or TeamViewer ${ }^{4}$ can also be used in the Android-based mobile domain.

\section{GlucoTab® Integration Scenarios}

Technical prerequisites may vary between different hospitals. Therefore, we attempt to provide five different integration scenarios for the GlucoTab ${ }^{\circledR}$ system.

\footnotetext{
${ }^{3}$ https://www.realvnc.com/products/android/

${ }^{4}$ http://www.teamviewer.com/en/download/mobile.aspx
}

\footnotetext{
${ }^{1}$ http://www.air-watch.com/

2 http://www.mobileiron.com/en/solutions/mobile-devicemanagement
} 
1) Basic Integration: This scenario has been implemented for the already performed clinical trials. The following integration steps have been achieved: (1) Installation of the GlucoTab ${ }^{\circledR}$ backend on a hospital server, (2) HL7 interface for automated patient management and (3) use of the hospital Wi-Fi for installation of the Samsung Galaxy Tab that provides the GlucoTab ${ }^{\circledR}$ frontend App.

2) Extended Integration: In the basic scenario we installed a user management developed in-house without a direct connection to the hospital user management. This resulted in an increased work load to keep patient management up-to-date. Moreover, in the basic scenario medical parameters like blood glucose and creatinine values had to been entered manually. The following extensions should be provided in the extended integration scenario to overcome these limitations. In addition to the basic integration scenario (4) an active directory interface should be implemented to directly use the hospital user management and (5) blood glucose values and other medical parameters like creatinine value should be transmitted via HL7 from the laboratory automatically if available.

3) Deep Integration I: If an electronic medical record (EMR) with software-based fever chart and CPOE is available in a hospital, GlucoTab ${ }^{\circledR}$ functionality should be integrated as deep as possible into the EMR using HL7 communication between GlucoTab ${ }^{\circledR}$ and the EMR based on the IHE profile "Hospital Medication Workflow (HMW)" [9].

4) Deep Integration II: If the EMR has been certified as a medical device, implementation of the GlucoTab ${ }^{\circledR}$ functionality directly in the EMR would be possible. In this scenario only one system would remain.

5) No Integration: If no integration is possible (technical prerequisites like Wi-Fi or HIS are not available) or an integration is not desired (e.g. during a pilot study) the following five requirements have to be met: (1) Hosting of the GlucoTab ${ }^{\circledR}$ server outside the hospital, (2) connection between GlucoTab ${ }^{\circledR}$ server and clients using a GSM network, (3) use of the already developed standalone user management, (4) manual management of patient transfers and (5) manual entry of medical parameters like blood glucose values.

\section{CONCLUSIONS}

The use of modern mobile devices like smartphones or tablets offers various advantages to HP but it also poses challenges to manufacturers and IT-operators. Based on our experiences from the GlucoTab ${ }^{\circledR}$ system we provided solutions how to integrate and operate mobile systems in hospitals.

\section{ACKNOWLEDGMENT}

We thank T. Truskaller, R. Moser, K. Pickl and S. Raudner (HEALTH - Institute for Biomedicine and Health Sciences, JOANNEUM RESEARCH GmbH, Graz, Austria) and G. Petritsch (Technical University of Graz, Austria) for ensuring a high software quality as part of the GlucoTab® development team. We also thank the Steiermärkische Krankenanstaltengesellschaft m.b.H. (KAGes) for supporting the integration process at the Medical University of Graz.

The development of the GlucoTab® system was partly funded by the European Commission under the 7th Framework Programme in the area of Personal Health Systems under Grant Agreement no. 248590 (REACTION project $\left.^{5}\right)$.

\section{REFERENCES}

mobiThinking, "Global mobile statistics 2014 Part A: Mobile subscribers; handset market share; mobile operators,” 2014. [Online]. Available: http://mobithinking.com/mobile-marketingtools/latest-mobile-stats/a\#subscribers. [Accessed: 28-Jul-2014].

K. Nirantharakumar, Y. F. Chen, T. Marshall, J. Webber, and J. J. Coleman, "Clinical decision support systems in the care of inpatients with diabetes in non-critical care setting: systematic review.,” Diabet. Med., vol. 29, no. 6, pp. 698-708, Jun. 2012.

N. J. Wei and D. J. Wexler, "Basal-bolus insulin protocols enter the computer age.,” Curr. Diab. Rep., vol. 12, no. 1, pp. 119-26, Feb. 2012 .

S. Spat, B. Höll, P. Beck, F. Chiarurgi, V. Kontogiannis, M. Spanakis, D. Manousos, and T. R. Pieber, “A Mobile AndroidBased Application for In-hospital Glucose Management in Compliance with the Medical Device Directive for Software,” in Wireless Mobile Communication and Healthcare, 2012, vol. 83, pp. 211-216.

B. Höll, S. Spat, J. Plank, L. Schaupp, K. Neubauer, P. Beck, F. Chiarugi, V. Kontogiannis, T. R. Pieber, and A. Holzinger, "Design of a Mobile, Safety-Critical in-Patient Glucose Management System.," Stud. Health Technol. Inform., vol. 169, pp. 31-36, 2011.

[6] S. Spat, B. Höll, G. Petritsch, L. Schaupp, P. Beck, and T. R. Pieber, "Automatic system testing of a decision support system for insulin dosing using Google Android.,” Stud. Health Technol. Inform., vol. 186, pp. 187-91, 2013.

[7] U. Buddrus, "mHEALTH - von IT zu Telemonitoring: Innovative Lösungsansätze,” 2013. [Online]. Available: www.ehealthsummit.at/sites/default/files/ehsat_mHealth_Buddrus.p df. [Accessed: 25-Jul-2014].

[8] HIMSS Analytics, "3rd Annual HIMSS Analytics Mobile Survey,” 2014.

IHE Pharmacy Technical Committee, "IHE Technical Framework Supplement - Hospital Medication Workflow (HMW),” 2013.

\footnotetext{
${ }^{5}$ http://www.reaction-project.eu/news.php
} 